\title{
Pathology findings in pediatric patients with COVID-19 and kidney dysfunction
}

\author{
Eric Nomura ${ }^{1}$ - Laura S. Finn ${ }^{2} \cdot$ Abbie Bauer $^{3} \cdot$ David Rozansky $^{3} \cdot$ Sandra Iragorri $^{3} \cdot$ Randall Jenkins $^{3} \cdot$ Amira Al-Uzri $^{3}$. \\ Kelsey Richardson ${ }^{3} \cdot$ Mary Wright $^{3} \cdot$ Vanderlene L. Kung $^{1} \cdot$ Megan L. Troxell ${ }^{4} \cdot$ Nicole K. Andeen $^{1}$
}

Received: 8 November 2021 / Revised: 4 January 2022 / Accepted: 4 January 2022 / Published online: 15 February 2022

(c) The Author(s), under exclusive licence to International Pediatric Nephrology Association 2022

\begin{abstract}
Background Acute kidney injury (AKI) is seen in one-fifth of pediatric patients with COVID-19 requiring hospital admission, and is associated with increased morbidity, mortality, and residual kidney impairment. The majority of kidney pathology data in patients with COVID-19 is derived from adult case series and there is an overall lack of histologic data for most pediatric patients with COVID-19.

Methods We assembled a multi-institutional cohort of five unvaccinated pediatric patients with COVID-19 and associated kidney dysfunction with available histology.

Results Three complex patients with current or prior SARS-CoV-2 infection had multifactorial thrombotic microangiopathy with clinical features of hemolytic uremic syndrome (in two) or disseminated intravascular coagulation (in one); one died and another developed chronic kidney disease stage 5. Two with recently preceding SARS-CoV-2 infection presented with nephrotic syndrome; one had IgA vasculitis and one had minimal change disease. Within a short follow-up time, none has returned to baseline kidney function.

Conclusion Although uncommon, COVID-19-associated kidney injury can have significant morbidity in the unvaccinated pediatric and adolescent population.
\end{abstract}

Keywords Kidney biopsy $\cdot$ Renal pathology $\cdot$ COVID-19 $\cdot$ SARS-CoV-2 $\cdot$ Kidney dysfunction $\cdot$ AKI $\cdot$ Acute kidney injury · Proteinuria $\cdot$ Nephrotic syndrome $\cdot$ TMA $\cdot$ Thrombotic microangiopathy $\cdot$ HUS $\cdot$ Hemolytic uremic syndrome $\cdot$

Glomerulonephritis $\cdot$ Henoch-Schönlein purpura $\cdot$ HSP $\cdot \operatorname{Ig}$ A vasculitis

\section{Background}

Severe acute respiratory syndrome coronavirus 2 (SARSCoV-2) infection and associated COVID-19 disease in the pediatric population have generally mild symptomatology and low mortality [1]. Acute kidney injury (AKI) is seen in

Nicole K. Andeen

andeen@ohsu.edu

1 Department of Pathology and Laboratory Medicine, Oregon Health \& Science University, 3181 SW Sam Jackson Park Road, Portland, OR 97239, USA

2 Department of Pathology, Children's Hospital of Philadelphia, Philadelphia, USA

3 Division of Pediatric Nephrology and Hypertension, Oregon Health \& Science University, Portland, USA

4 Department of Pathology, Stanford University, Stanford, USA one-fifth of pediatric patients requiring hospital admission, and is associated with increased morbidity, mortality, and residual kidney impairment [2-4]. The majority of kidney pathology data in patients with COVID-19 is derived from adult case series; investigators have described pathologic findings in a few recent case reports [5-9], but there is an overall lack of histologic data for most pediatric patients. We sought to better understand the histopathology and clinical findings in pediatric patients with COVID-19-associated kidney dysfunction.

\section{Methods}

This study was approved by the Institutional Review Boards (IRB) and adheres to the Declaration of Helsinki. Native kidney pathology databases were searched from 2020 to 2021 for pediatric patients $\leq 18$ years of age with 
COVID-19 and available kidney pathology at Oregon Health and Science University and Doernbecher Children's Hospital, Stanford University and Lucile Packard Children's Hospital, and Children's Hospital of Philadelphia. Biopsies had standard pathologic workup, including light microscopic evaluation with Jones methenamine silver, periodic acid Schiff (PAS), hematoxylin and eosin (H\&E), and trichrome stains. For immunofluorescence (IF) microscopy, frozen tissue was stained with antibodies against IgG, IgA, IgM, C3, C1q, fibrin/fibrinogen, and kappa and lambda light chains. Electron microscopic (EM) evaluation followed standard protocols. Clinical history was obtained through discussion with nephrologists and review of the medical record. Coronavirus-like particles were not seen on EM, and SARS-CoV-2 tissue testing was not performed on any of the kidneys as we and others have not identified SARS-CoV-2 in kidney biopsy tissue in previous studies [10-12].

\section{Results}

Five pediatric patients with COVID-19 and associated kidney dysfunction had kidney histology available (4 kidney biopsies, 1 autopsy), performed between December 2020 and September 2021. None had been vaccinated against COVID-19. During this time period, the cumulative incidence of COVID-19-associated hospitalizations in children was approximately 49.7 per 100,000 (March 2020-August 2021) [13]; weekly hospitalization rates varied 5-tenfold by time period, age group, and vaccination status. With the emergence of the Delta variant, higher rates of hospitalization, need for invasive mechanical ventilation, and death were observed during the summer of 2021 [13]. During this time period, there were no approved COVID-19 vaccines in the USA for children under the age of 12. Children aged $\geq 12$ years were eligible to receive Pfizer-BioNTech COVID-19 vaccine prior to the emergence of the Delta variant in May 2021. Although viral genotyping was not performed, cases 2 and 5 occurred during winter/spring of 2020-21, during which Alpha and Epsilon were the dominant SARS-CoV-2 variants in the USA. Cases 1, 3, and 4 occurred during summer/fall 2021, during which time Delta was the dominant SARS-CoV-2 variant.

The cohort included 3 males and 2 females aged 5 to 18 years; 3 presented with AKI and had systemic features of thrombotic microangiopathy (TMA), hemolytic uremic syndrome (HUS), or disseminated intravascular coagulation (DIC). Two presented with nephrotic syndrome, which was accompanied by severe abdominal pain and hematochezia in one. Clinical and pathologic findings are summarized by case below, and in Table 1.

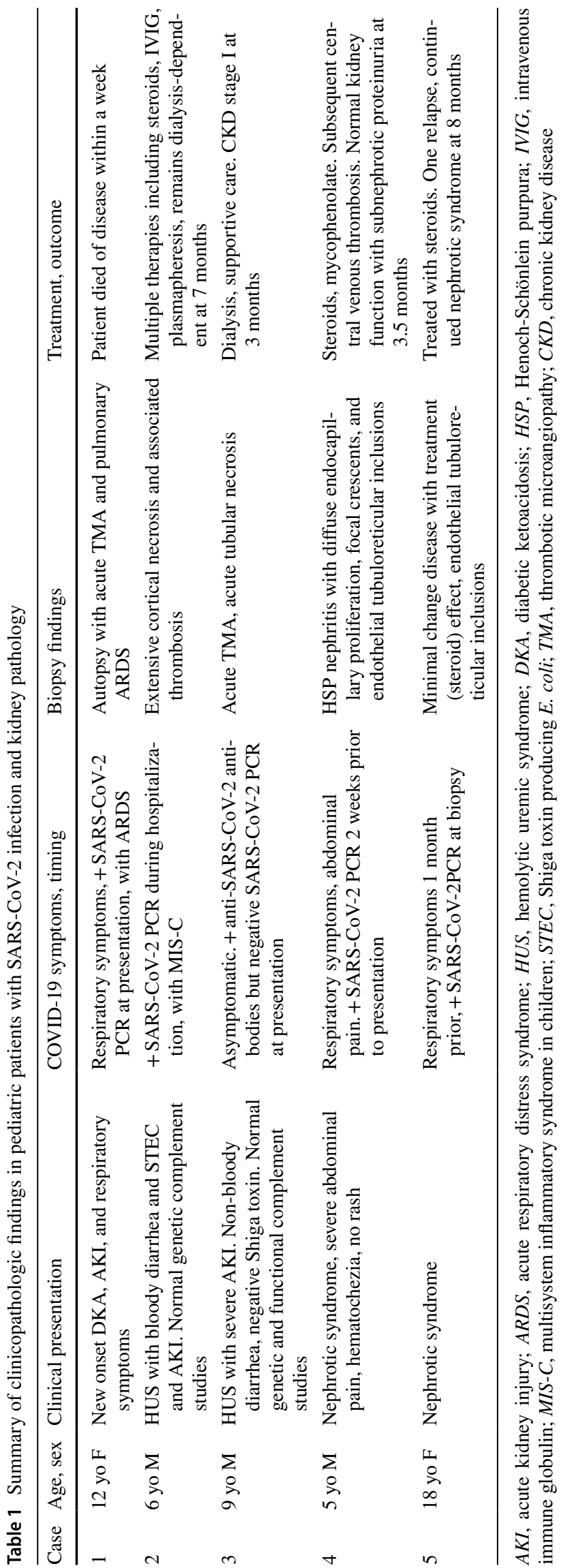




\section{Case 1}

A 12-year-old White female with a history of autism, obstructive sleep apnea, and recurrent urinary tract infections presented with new onset diabetic ketoacidosis (DKA) and altered mental status after a few days of fever, malaise, cough, and chest pain. She was found to have a positive SARS-CoV-2 PCR, and chest X-ray showed pneumonia. After initial response to DKA therapy, she developed worsening respiratory distress and was intubated, subsequently suffering 3 cardiac arrests and was placed on ECMO. Over the following few days, she experienced persistent COVID19 acute respiratory distress syndrome (ARDS) with refractory hypoxia, myocardial dysfunction, vasopressor refractory shock, altered mental status, AKI, DIC, and marked electrolyte abnormalities (severe hypophosphatemia and hypokalemia). Due to concern for neurologic sequela from multiple cardiac arrests, the family elected comfort care and the patient died a few days after initial presentation. Postmortem evaluation revealed TMA involving glomerular capillaries (Fig. 1A) and histologic features of ARDS with pulmonary alveolar hyaline membranes, pulmonary parenchymal necrosis, and arterial thromboses. Staphylococcus aureus grew from post-mortem lung cultures.

\section{Case 2}

A 6-year-old Hispanic male with no significant past medical history presented with a 3-day history of abdominal pain, emesis, and hematochezia. He had initially normal kidney function (serum creatinine $(\mathrm{Cr}) 0.5 \mathrm{mg} / \mathrm{dL}$ ) (reference range $0.3-0.7 \mathrm{mg} / \mathrm{dL}$ ) and platelet count, but quickly developed anemia (hemoglobin $11 \mathrm{~g} / \mathrm{dL}$ ) (reference range 11.5-15.5 g/dL), thrombocytopenia (platelets 35,000/
uL) (reference range 150,000-440,000/uL), and AKI (Cr $3.4 \mathrm{mg} / \mathrm{dL}$ ). Stool was positive for Shiga toxin producing Escherichia coli (STEC), leading to a diagnosis of Shiga toxin diarrhea + HUS. He was admitted and developed respiratory distress, SARS-CoV-2 infection, and later multisystem inflammatory syndrome in children (MIS-C) with cardiac involvement manifested as dilatation of left main, left anterior descending, and right coronary arteries, and biatrial dilatation with pericardial effusion.

During a prolonged hospitalization and pediatric intensive care unit (PICU) stay, the patient was treated with pulse solumedrol ( $30 \mathrm{mg} / \mathrm{kg} \times 3$ days), intravenous immune globulin (IVIg), plasmapheresis (5 rounds), and dialysis. Kidney biopsy performed due to lack of kidney recovery demonstrated extensive cortical necrosis and thrombosis involving the entire biopsy core with all sampled vasculature including glomeruli, arterioles, and interlobular arteries (Fig. 1B). Given severity of his clinical course, genetic testing was performed and was negative for abnormalities of the alternative complement pathway (in ADAMTS13, C2, C3, C3AR1, CD46 (MCP), CFB, CFD, CFH, CFHR1, CFHR2, CFHR3, CFHR4, CFHR5, CFI, DGKE, MASP2, MMACHC, $T H B D, P L G, W T 1$, and $C 5$ p.Arg885, Machaon Diagnostics). The extent of renal cortical necrosis and thrombosis was considered likely due to a combination of Shiga toxin diarrhea + HUS and COVID-19 with associated MIS-C. At 7 months, echocardiogram demonstrated normalization of cardiac abnormalities, but he remained dialysis-dependent and was undergoing evaluation for kidney transplant.

\section{Case 3}

A 9-year-old White male, previously healthy, who had not received any typical childhood vaccines presented with 2 weeks of fever, nausea, and non-bloody diarrhea. On
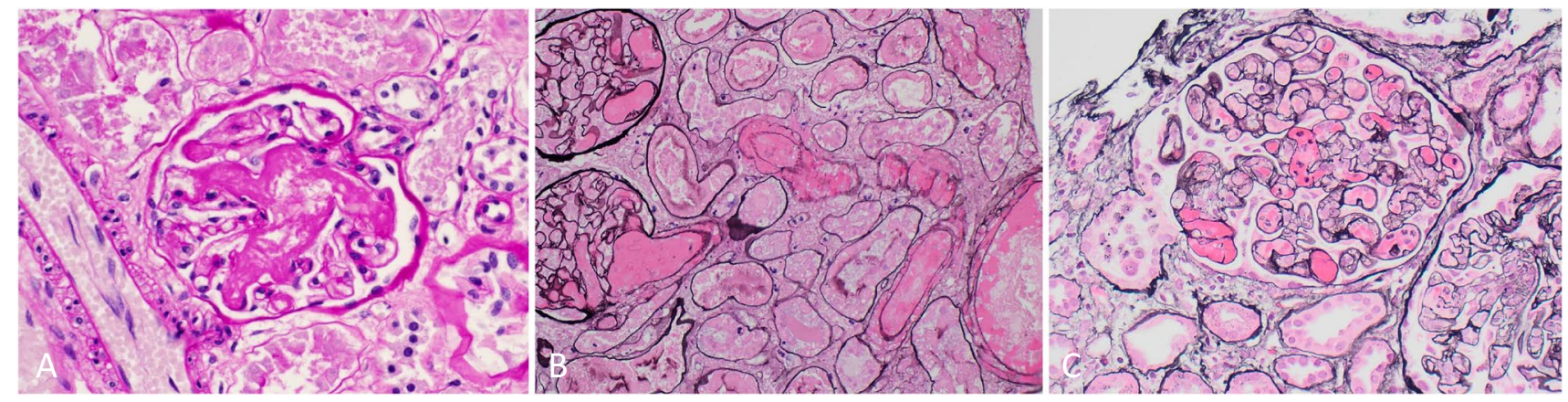

Fig. 1 Kidneys with thrombotic microangiopathy in the setting of current or prior COVID-19, with (A) glomerular mesangiolysis and fibrin thrombi in an autopsy from a patient with disseminated intravascular coagulation (case 1 PAS $\times 200$ ), (B) extensive cortical necrosis with intravascular thromboses (case 2, Jones $\times 100$ ), and $(\mathbf{C})$ mesangiolysis, fibrin thrombi, and adjacent acute tubular injury (case 3 , Jones $\times 200$ ) 
presentation, he was hypertensive (136/86) and laboratory studies were concerning for HUS, with hemoglobin of $9.3 \mathrm{~g} / \mathrm{dL}$ platelets $148,000 / \mathrm{uL}, \mathrm{LDH} 1835 \mathrm{U} / \mathrm{L}$ (reference range $\leq 350 \mathrm{U} / \mathrm{L}$ ), and schistocytes on blood smear. He had AKI with $\mathrm{Cr}$ of $20.9 \mathrm{mg} / \mathrm{dL}$, BUN of $256 \mathrm{mg} / \mathrm{dL}$ (reference range $8-25 \mathrm{mg} / \mathrm{dL}$ ), hematuria, and nephrotic range proteinuria with a urine protein to creatinine ratio (UPCR) of $8.07 \mathrm{mg} / \mathrm{mg}$ (reference range $<0.10 \mathrm{mg} / \mathrm{mg}$ ). PCR for SARS-CoV-2 was negative but COVID-19 antibodies were detected at admission, with unknown time of infection. Infectious workup was otherwise negative including stool studies which were negative for Shiga toxins 1 and 2, Shigella, and enteropathogenic viruses and bacteria, and negative hepatitis B and anti-DNase B.

A kidney biopsy was performed due to concern for TMA, and demonstrated acute TMA and acute tubular necrosis (Fig. 1C). Results of a TMA functional panel including CH50 level, alternative pathway functional assay, $\mathrm{C} 3 \mathrm{~b}$ deposition, factor $\mathrm{H}$ autoantibody, C3 level, $\mathrm{C} 4$ level, factor B, Bb level, soluble C5b-9 (sMAC), factor $\mathrm{H}$ level, and factor I levels were all within normal limits (University of Iowa). Genetic testing for abnormalities associated with atypical HUS was negative for clinically significant mutations (Machaon Diagnostics). Thus, despite extensive workup, a definitive etiology for the episode of HUS was not identified, with prior SARSCoV-2 infection representing a potential trigger. He was treated with inpatient hemodialysis, supportive care, and anti-hypertensives (amlodipine, isradipine as needed) without immunosuppressants. At 6 weeks follow-up, kidney function, proteinuria, and TMA labs had improved but he had persistent renal insufficiency $(\mathrm{Cr} 0.9 \mathrm{mg} / \mathrm{dL})$ with subnephrotic proteinuria (UPCR $1.02 \mathrm{mg} / \mathrm{mg}$ ). At 3 months follow-up, $\mathrm{Cr}$ improved to $0.6 \mathrm{mg} / \mathrm{dL}$ (eGFR $100 \mathrm{~mL} / \mathrm{min} 1.73 \mathrm{~m}^{2}$ ), considered chronic kidney disease (CKD) stage I.

\section{Case 4}

A 5-year-old, White male, previously healthy, presented with cough and was found to have a positive SARS-CoV-2 PCR. Approximately 2 weeks later, he experienced progressive, severe abdominal pain with palpable purpura and petechial rash, hematochezia, microscopic hematuria, and nephrotic syndrome with hypoalbuminemia $(2.1 \mathrm{~g} / \mathrm{dL}$, reference range $3.5-5.5 \mathrm{~g} / \mathrm{dL}$ ), proteinuria (UPCR $9.88 \mathrm{mg} /$ $\mathrm{mg}$ ), hypertension, and edema with weight gain (10 lbs.) with a normal creatinine $(0.29 \mathrm{mg} / \mathrm{dL})$. Due to concern for Henoch-Schönlein purpura (HSP) nephritis/IgA vasculitis following COVID-19, a kidney biopsy was performed and revealed HSP nephritis/IgA vasculitis with diffuse endocapillary hypercellularity, focal crescents $(5 \%)$, moderate podocyte foot process effacement, and endothelial tubuloreticular inclusions (Fig. 2). He was treated with IV pulse steroids (methylprednisolone $30 \mathrm{mg} / \mathrm{kg} /$ day) followed by oral steroids (prednisolone $2 \mathrm{mg} / \mathrm{kg}$ /day) with weekly solumedrol infusions $(30 \mathrm{mg} / \mathrm{kg})$; mycophenolate mofetil followed by enalapril was subsequently added due to ongoing proteinuria. Nephrotic syndrome was managed with $25 \%$ albumin and furosemide. A few days after discharge, he was readmitted with severe headache and found to have a cerebral venous sinus thrombosis, which was treated with enoxaparin. He continued to have nephrotic range proteinuria (UPCR $3.79 \mathrm{mg} / \mathrm{mg}$ ) at 6 weeks, and had multiple readmissions for severe headaches. At 3.5 months of follow-up, headaches had improved, $\mathrm{Cr}$ remained within normal limits, and proteinuria had decreased to $1.4 \mathrm{mg} / \mathrm{mg}$.

\section{Case 5}

An 18-year-old Hispanic female with no significant past medical history presented with swelling and was found to have nephrotic syndrome with hypoalbuminemia $(2.2 \mathrm{~g} /$
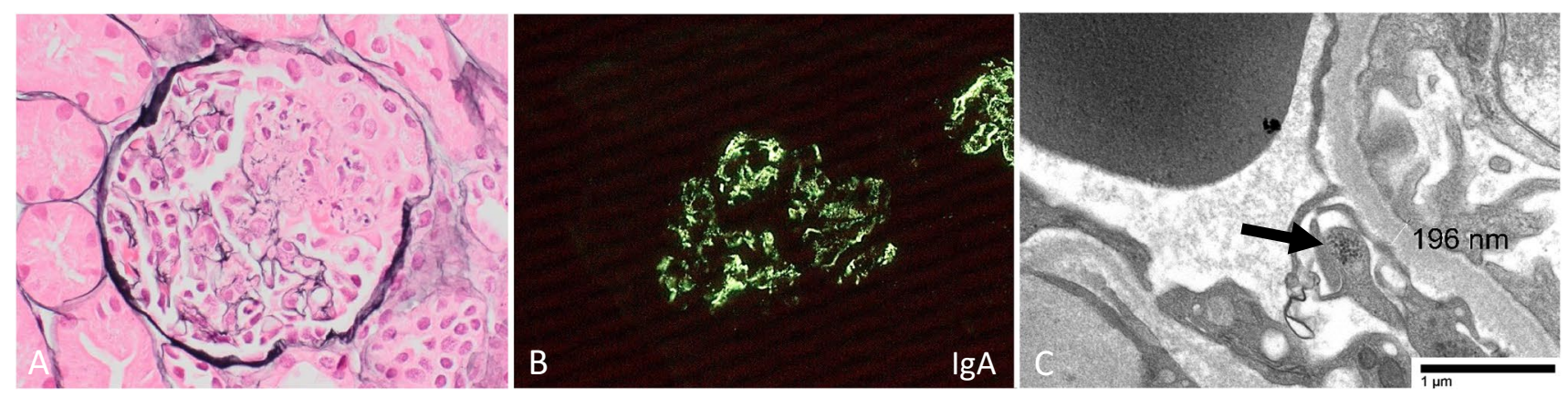

Fig. 2 Henoch-Schönlein purpura nephritis/IgA vasculitis following SARS-CoV-2 infection, with (A) focal crescents (Jones $\times 400$ ), (B) predominantly mesangial deposition of $\operatorname{IgA}$, and (C) endothelial tubuloreticular inclusions (arrow, transmission electron microscopy, direct magnification $\times 4800$ ) 
$\mathrm{dL}$ ), hyperlipidemia (total cholesterol $405 \mathrm{mg} / \mathrm{dL}$, reference range $<200 \mathrm{mg} / \mathrm{dL}$ ), and $7.79 \mathrm{~g}$ of proteinuria on calculated 24-h urine. She was positive for SARS-CoV-2 by PCR and reported respiratory symptoms 1 month prior which had resolved by the time of presentation for edema. Other laboratory tests including antinuclear antibodies (ANA) and complement levels were negative/normal. She was treated with prednisone $(60 \mathrm{mg} /$ day $)$. A subsequent kidney biopsy performed to determine histologic diagnosis for apparently steroid sensitive nephrotic syndrome demonstrated mild, patchy podocyte injury, and scattered tubuloreticular inclusions, consistent with minimal change disease with treatment effect (Fig. 3). Although proteinuria initially resolved after 2 weeks of steroid therapy, at 8 months post-biopsy, the patient had experienced one relapse and had nephrotic syndrome (albumin $2.1 \mathrm{~g} / \mathrm{dL}$, calculated $24-\mathrm{h}$ proteinuria $4.62 \mathrm{~g}$ ) at last follow-up.

\section{Discussion}

We present a multi-institutional cohort of five pediatric patients with COVID-19 and associated kidney dysfunction with available histology. In our series, SARS-CoV-2 infection in children ranged from clinically asymptomatic to severe with MIS-C and ARDS. Kidney pathology demonstrated TMA in three patients with AKI requiring hospitalization. Two had nephrotic syndrome, one with immune complex disease (HSP nephritis), and one with a podocytopathy (minimal change disease). This broad spectrum of kidney injury in the setting of COVID-19 is similar to that reported in the adult population [10-12, 14-17]. Within a short follow-up time, one patient died and the remaining four are currently being treated and have not returned to baseline kidney function.

Three patients with current or prior COVID-19 presented with AKI and systemic features of acute endothelial injury in the spectrum of HUS or DIC. Neither of the two tested patients had underlying abnormalities of the alternative complement pathway. The first (case 1) experienced
COVID-19-related ARDS and DIC in the setting of new onset DKA. DKA is known to be precipitated by infections, and COVID-19 represents an additional infection to consider in newly diagnosed diabetics presenting with DKA. At least one had another reason for TMA (STEC HUS, case 2), but given the concurrent SARS-CoV-2 infection, MIS-C, and extensive renal cortical necrosis with resultant chronic kidney disease stage 5, COVID-19 was considered a likely contributor to the severity of the TMA and clinical course [5, 18, 19]. COVID-19-associated TMA has been described in pediatric patients [19], including at least one patient with a kidney biopsy who had MIS-C with coronary artery dilatation ("Kawasaki-like" disease), similar to ours [5]. The third (case 3) had no definitive etiology (Shiga toxin negative diarrhea); although COVID-19 antibodies were detected at presentation, the timing of prior SARS-Cov- 2 infection was unknown. Given the presence of COVID-19 antibodies and reports of TMA after SARS-CoV-2 infection in children [5, 19] and adults [10, 14], this represents a potential but unproven trigger for TMA. There is uncertainty as to whether SARS-CoV-2 is a cause of, or contributor to, TMA in these three cases, particularly cases 2 and 3 . These cases and their complexity bear similarities to adult series of TMA in the setting of COVID-19, which have been reported predominantly in patients with underlying conditions and are likely mediated by multifactorial endothelial injury [20,21]. Future studies may help elucidate associations and biologic mechanisms determining development of TMA in children with SARS-CoV-2 infection.

Two previously healthy patients presented with nephrotic syndrome shortly after SARS-CoV-2 infection: one had HSP nephritis/IgA vasculitis, and one had minimal change disease. Both of these biopsies contained endothelial tubuloreticular inclusions, a recognized morphologic manifestation of a high-interferon state seen in the setting of COVID-19 and other viral and autoimmune diseases, particularly untreated hepatitis $\mathrm{B}$ or $\mathrm{C}$ (HBV, HCV), human immunodeficiency virus (HIV), and lupus. Pediatric IgA vasculitis after COVID-19 has been described in at least 3 case reports $[6,7,22]$. Notably,
Fig. 3 Minimal change disease following SARS-CoV-2 infection, with (A) normal glomeruli by light microscopy $(\mathrm{PAS} \times 400)$, and $($ B $)$ ultrastructural studies demonstrating endothelial tubuloreticular inclusions (arrow) and predominantly intact podocyte foot processes consistent with steroid treatment effect (transmission electron microscopy, direct magnification $\times 7000$ )

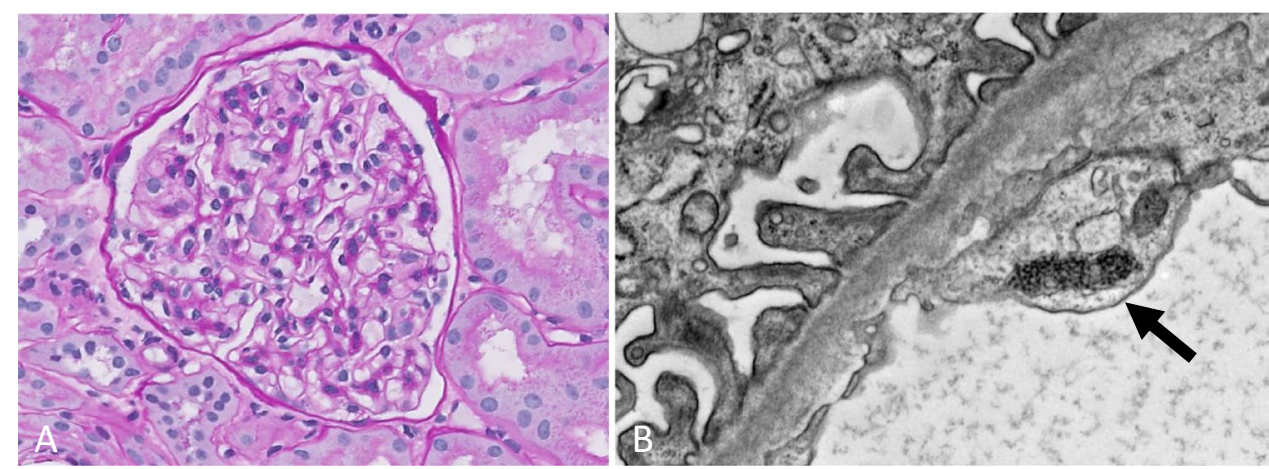


post-COVID-19 IgA nephropathy/vasculitis is comparatively under-reported in adult kidney histology-based case series, in which the most common glomerular finding is collapsing glomerulopathy-usually associated with APOL1 high-risk alleles-in addition to acute tubular injury [12]. However, clinically apparent and biopsyproven flares of IgA nephropathy and IgA vasculitis have been described in adults after SARS-CoV-2 mRNA vaccination [23-26], suggesting an immunologic connection between SARS-CoV-2 and IgA nephropathy/vasculitis in both adults and children, which deserves further study. Similar to our case, post-COVID-19, steroid-responsive nephrotic syndrome consistent with minimal change disease has also previously been reported in a 5-year-old girl (not biopsied) [27], and relapse of nephrotic syndrome after COVID-19 has also been reported [28]. This highlights the apparent podocytopathic effects of SARS-CoV-2 in addition to its known endothelial tropism and capacity for systemic immune activation.

In addition to kidney biopsy findings seen in our series, post-COVID-19 tubulointerstitial nephritis in two children [6] including one with uveitis (TINU) [29] has previously been reported. COVID-19-associated crescentic glomerulonephritis in 2 pediatric patients has also been documented as has de novo collapsing glomerulopathy in a kidney transplant patient [9], but were not present in our cohort. Kidney injury and biopsy findings following COVID-19 infection in children and adolescents have recently been reviewed elsewhere [30]. The proposed mechanisms of COVID-19-associated kidney injury include immune system dysregulation, cytokine activation, dysfunction of coagulation, complement of the microcirculatory system, and some pathophysiology shared with sepsis-associated AKI [31-34]. In our cohort, two patients (cases 1 and 2) presented acutely, and their AKI and TMA were likely driven by more than one of these proposed mechanisms. Three patients (cases 3, 4, and 5) experienced only mild COVID-19 symptoms which preceded kidney dysfunction, suggesting that SARS-CoV-2 infection could have precipitated subsequent immune dysregulation, albeit with morphologically diverse manifestations.

\section{Conclusion}

In summary, we describe five pediatric patients with COVID-19-associated kidney dysfunction, manifesting as TMA, HSP nephritis/IgA vasculitis, and minimal change disease. Although uncommon, COVID-19-associated kidney injury can have significant morbidity in the unvaccinated pediatric and adolescent population.
Supplementary Information The online version contains supplementary material available at https://doi.org/10.1007/s00467-022-05457-w.

\section{Declarations}

Ethics approval This study is approved by our Institutional Review Board (IRB).

Consent to participate Not applicable.

Consent for publication Not applicable.

Conflict of interest The authors declare no competing interests.

\section{References}

1. Badal S, ThapaBajgain K, Badal S, Thapa R, Bajgain BB, Santana MJ (2021) Prevalence, clinical characteristics, and outcomes of pediatric COVID-19: a systematic review and meta-analysis. J Clin Virol 135:104715

2. Kari JA, Shalaby MA, Albanna AS, Alahmadi TS, Alherbish A, Alhasan KA (2021) Acute kidney injury in children with COVID19: a retrospective study. BMC Nephrol 22:202

3. Wang X, Chen X, Tang F, Luo W, Fang J et al (2021) Be aware of acute kidney injury in critically ill children with COVID-19. Pediatr Nephrol 36:163-169

4. Basalely A, Gurusinghe S, Schneider J, Shah SS, Siegel LB et al (2021) Acute kidney injury in pediatric patients hospitalized with acute COVID-19 and multisystem inflammatory syndrome in children associated with COVID-19. Kidney Int 100:138-145

5. Oi SSP, Muniz MPR, Faria IM, Filho NS, de Brito DJA et al (2021) Multisystemic inflammatory syndrome and thrombotic microangiopathy as complications of COVID-19 in a child: a case report. Front Pediatr 9:659069

6. Serafinelli J, Mastrangelo A, Morello W, Cerioni VF, Salim A et al (2021) Kidney involvement and histological findings in two pediatric COVID-19 patients. Pediatr Nephrol 36:3789-3793

7. AlGhoozi DA, AlKhayyat HM (2021) A child with HenochSchonlein purpura secondary to a COVID-19 infection. BMJ Case Rep 14:e239910

8. Basiratnia M, Derakhshan D, Yeganeh BS, Derakhshan A (2021) Acute necrotizing glomerulonephritis associated with COVID19 infection: report of two pediatric cases. Pediatr Nephrol 36:1019-1023

9. Levenson E, Shepherd TN, Aviles D, Craver R, Ehlayel A et al (2021) De novo collapsing glomerulopathy in a pediatric kidney transplant recipient with COVID-19 infection. Pediatr Transplant 25:e14013

10. Akilesh S, Nast CC, Yamashita M, Henriksen K, Charu V et al (2021) Multicenter clinicopathologic correlation of kidney biopsies performed in COVID-19 patients presenting with acute kidney injury or proteinuria. Am J Kidney Dis 77:82-93

11. Kudose S, Batal I, Santoriello D, Xu K, Barasch J et al (2020) Kidney biopsy findings in patients with COVID-19. J Am Soc Nephrol 31:1959-1968

12. May RM, Cassol C, Hannoudi A, Larsen CP, Lerma EV et al (2021) A multi-center retrospective cohort study defines the spectrum of kidney pathology in Coronavirus 2019 Disease (COVID19). Kidney Int 100:1303-1315

13. Delahoy MJ, Ujamaa D, Whitaker M, O'Halloran A, Anglin O et al (2021) Hospitalizations associated with COVID-19 among 
children and adolescents - COVID-NET, 14 States, March 1, 2020-August 14, 2021. MMWR Morb Mortal Wkly Rep 70:1255-1260

14. Sharma P, Uppal NN, Wanchoo R, Shah HH, Yang Y et al (2020) COVID-19-associated kidney injury: a case series of kidney biopsy findings. J Am Soc Nephrol 31:1948-1958

15. Santoriello D, Khairallah P, Bomback AS, Xu K, Kudose S et al (2020) Postmortem kidney pathology findings in patients with COVID-19. J Am Soc Nephrol 31:2158-2167

16. Su H, Yang M, Wan C, Yi LX, Tang F et al (2020) Renal histopathological analysis of 26 postmortem findings of patients with COVID-19 in China. Kidney Int 98:219-227

17. Nasr SH, Alexander MP, Cornell LD, Herrera LH, Fidler ME et al (2021) Kidney biopsy findings in patients with COVID-19, kidney injury, and proteinuria. Am J Kidney Dis 77:465-468

18. Grewal MK, Gregory MJ, Jain A, Mohammad D, Cashen K et al (2021) Acute kidney injury in pediatric acute SARS-CoV-2 infection and multisystem inflammatory syndrome in children (MISC): is there a difference? Front Pediatr 9:692256

19. Diorio C, McNerney KO, Lambert M, Paessler M, Anderson EM et al (2020) Evidence of thrombotic microangiopathy in children with SARS-CoV-2 across the spectrum of clinical presentations. Blood Adv 4:6051-6063

20. Teuwen LA, Geldhof V, Pasut A, Carmeliet P (2020) COVID-19: the vasculature unleashed. Nat Rev Immunol 20:389-391

21. Ortega-Paz L, Capodanno D, Montalescot G, Angiolillo DJ (2021) coronavirus disease 2019-associated thrombosis and coagulopathy: review of the pathophysiological characteristics and implications for antithrombotic management. J Am Heart Assoc 10:e19650

22. El Hasbani G, Taher AT, Jawad ASM, Uthman I (2021) HenochSchonlein purpura: another COVID-19 complication. Pediatr Dermatol 38:1359-1360

23. Negrea L, Rovin BH (2021) Gross hematuria following vaccination for severe acute respiratory syndrome coronavirus 2 in 2 patients with IgA nephropathy. Kidney Int 99:1487

24. Park K, Miyake S, Tai C, Tseng M, Andeen NK, Kung VL (2021) Letter regarding: a case of gross hematuria and IgA nephropathy flare-up following SARS-CoV-2 vaccination. Kidney Int Rep 6:2246-2247
25. Rahim SEG, Lin JT, Wang JC (2021) A case of gross hematuria and IgA nephropathy flare-up following SARS-CoV-2 vaccination. Kidney Int 100:238

26. Bomback AS, Kudose S, D'Agati VD (2021) De novo and relapsing glomerular diseases after COVID-19 vaccination: what do we know so far? Am J Kidney Dis 78:477-480

27. Morgan KM, Imani PD (2021) Case report: a 5-year-old with new onset nephrotic syndrome in the setting of COVID-19 infection. BMC Nephrol 22:323

28. Basalely A, Brathwaite K, Duong MD, Liu D, Mazo A et al (2021) COVID-19 in children with kidney disease: a report of 2 cases. Kidney Med 3:120-123

29. Paladini A, Venturoli V, Mosconi G, Zambianchi L, Serra L, Valletta E (2013) Tubulointerstitial nephritis and uveitis syndrome in a twelve-year-old girl. Case Rep Pediatr 2013:652043

30. Wu HHL, Shenoy M, Kalra PA, Chinnadurai R (2022) Intrinsic kidney pathology following COVID-19 infection in children and adolescents: a systematic review. Children 9:3

31. Bjornstad EC, Seifert ME, Sanderson K, Feig DI (2021) Kidney implications of SARS-CoV2 infection in children. Pediatr Nephrol. https://doi.org/10.1007/s00467-021-05249-8

32. Legrand M, Bell S, Forni L, Joannidis M, Koyner JL et al (2021) Pathophysiology of COVID-19-associated acute kidney injury. Nat Rev Nephrol 17:751-764

33. Nadim MK, Forni LG, Mehta RL, Connor MJ Jr, Liu KD et al (2020) COVID-19-associated acute kidney injury: consensus report of the 25th Acute Disease Quality Initiative (ADQI) Workgroup. Nat Rev Nephrol 16:747-764

34. Faour WH, Choaib A, Issa E, Choueiry FE, Shbaklo K et al (2021) Mechanisms of COVID-19-induced kidney injury and current pharmacotherapies. Inflamm Res 71:39-56

Publisher's note Springer Nature remains neutral with regard to jurisdictional claims in published maps and institutional affiliations. 